\title{
Tuberculosis cutánea recurrente, variedad nodular profunda. Comunicación de un caso y revisión de la literatura
}

\author{
Cutaneous tuberculosis, deep nodular variety, \\ recurrent. Case report and review of the literature
}

\author{
Miguel Ángel Cardona-Hernández, ${ }^{*}$ Maribet González-González, ${ }^{\ddagger}$ \\ Fermín Jurado-Santa Cruz, ${ }^{\S}$ Ana Karen Romero-Guzmán"
}

\section{RESUMEN}

Se considera a la tuberculosis nodular profunda como una tuberculíde. Su diagnóstico se basa en las características clínicas, prueba de tuberculina positiva, evidencia de $M$. tuberculosis en otra localización a nivel sistémico y a nivel histopatológico, así como a la presencia de paniculitis lobulillar granulomatosa. En este artículo se comunica el caso de una mujer de 45 años de edad con diagnóstico de tuberculosis nodular profunda, quien inicialmente mejoró con la administración de terapia con antifímicos convencionales, con recurrencia del cuadro al término del tratamiento. Se realiza una revisión bibliográfica al respecto.

Palabras clave: Tuberculosis nodular profunda, tuberculíde, recurrencia.
ABSTRACT

Deep nodular tuberculosis is considered a tuberculide. Its diagnosis is based on the clinical characteristics, a positive tuberculin test, evidence of $M$. tuberculosis in another body site and, at the histopathological level, the presence of granulomatous lobular panniculitis. In this article we report the case of a 45-year-old woman with deep nodular tuberculosis, who initially improved with the administration of conventional antifimic therapy, with recurrence of the condition at the end of treatment. A bibliographic review is carried out in this regard.

Keywords: Deep nodular tuberculosis, tuberculide, recurrence.

\section{INTRODUCCIÓN}

La tuberculosis continúa siendo una de las principales causas de morbimortalidad a nivel mundial. De acuerdo con las cifras proporcionadas por la Organización Mundial de la Salud, ocupa el noveno lugar, por arriba del virus de la inmunodeficiencia humana (VIH). EI incremento en su incidencia mundial se debe a la propagación de la infección por $\mathrm{VIH}$, al desarrollo de cepas

\footnotetext{
* Dermatólogo.

‡ Dermatopatóloga.

$\S$ Responsable de la Unidad Médica.

" Residente de tercer año.
}

Centro Dermatológico «Dr. Ladislao de la Pascua», SSCDMX. México. multirresistentes y al empleo de inmunosupresores para tratar otras enfermedades. Es ocasionada por el Mycobacterium tuberculosis, el cual, dependiendo de la inmunocompetencia de cada individuo, de los factores ambientales y del tipo y cantidad del inóculo, ocasiona la enfermedad y se manifiesta en diferentes formas clínicas. El involucro cutáneo es poco frecuente, representa solamente $1-2 \%$ de todas las manifestaciones extrapulmonares. ${ }^{1,2}$

Para su clasificación se toman en cuenta tres criterios que engloban una amplia variedad de manifestaciones

Citar como: Cardona-Hernández MÁ, González-González M, Jurado-Santa Cruz F, Romero-Guzmán AK. Tuberculosis cutánea recurrente, variedad nodular profunda. Comunicación de un caso y revisión de la literatura. Rev Cent Dermatol Pascua. 2021; 30 (1): 34-38. https://dx.doi.org/10.35366/100590 
clínicas: patogénesis, presentación clínica y evaluación histopatológica. Con los criterios antes señalados, la tuberculosis cutánea (TBC) en la actualidad se clasifica de la siguiente manera: ${ }^{1}$

- Tuberculosis de fuente exógena: chancro tuberculoso, tuberculosis verrugosa.

- Tuberculosis de fuente endógena: escrofulodermia, tuberculosis orofacial.

- Tuberculosis hematógena: lupus vulgar, tuberculosis ganglionar, tuberculosis miliar, tuberculídes.

Anteriormente, la TBC se dividía en dos grandes grupos: TBC verdadera y las tuberculídes. Estas últimas son el resultado de una reacción de hipersensibilidad hacia los antígenos de $M$. tuberculosis; se clasifican en tuberculídes papulonecróticas, liquen escrofuloso y eritema indurado de Bazin..$^{3-5}$

La tuberculosis nodular profunda, también conocida como «eritema indurado de Bazin», fue descrita por Bazin en 1861. Se le conoce como una variedad casi exclusiva de las mujeres, de localización única en las piernas, sobre todo en sus caras posteriores, en forma bilateral, con tendencia a la simetría, caracterizada por la presencia de nódulos profundos, eritemato-violáceos, dolorosos, recurrentes y que en su involución dejan zonas atróficas deprimidas que deforman poco a poco las piernas. En algunas ocasiones tienden a ulcerarse en el centro hasta en $30 \%$, a este tipo de casos se les denomina "variedad tipo Hutchinson». ${ }^{3}$ La piel afectada se torna de color rojo, marrón o púrpura. Puede asociarse con insuficiencia venosa crónica, livedo reticularis, edema, eritrocianosis y queratosis folicular. ${ }^{4}$

Los brotes se presentan sobre todo durante el invierno, y mientras unas lesiones inician su evolución otras están terminándola, por lo que es posible observar lesiones en diferentes estadios de evolución.

Las lesiones nodulares generalmente se resuelven espontáneamente en unos cuantos meses y quedan como secuelas hiperpigmentación postinflamatoria y cicatrices atróficas. En aquellos casos con recaídas frecuentes puede desarrollarse linfedema.

El diagnóstico diferencial debe realizarse principalmente con eritema nudoso, poliarteritis nodosa, paniculitis, lupus profundo, sarcoidosis y linfoma paniculítico. ${ }^{5}$

La histopatología muestra infiltrados que ocupan dermis e hipodermis constituidos por linfocitos, histiocitos, células epitelioides y células gigantes multinucleadas de tipo Langhans, que se organizan para formar granulomas tuberculoides. Se acompañan de vasculitis que afecta vénulas y arterias de pequeño a mediano calibre, así como de necrosis caseosa. ${ }^{6}$

El diagnóstico de TBC se basa en la sospecha clínica, en los hallazgos del estudio histopatológico y de los exámenes de laboratorio. ${ }^{4}$ Los métodos empleados para detectar el agente causal incluyen cultivo de Lowenstein-Jensen (estándar de oro para el diagnóstico de TBC verdadera). Las pruebas para evaluar la inmunidad hacia la infección, como la tuberculina y el ensayo de liberación de interferón gamma (IGRA) son útiles para apoyar el diagnóstico, sobre todo cuando los resultados de la histopatología y microbiología son negativos o no concluyentes. Las pruebas de amplificación de ácidos nucleicos para detectar ADN del microorganismo corroboran el diagnóstico. ${ }^{7}$

En pacientes en quienes se tenga una fuerte sospecha clínica de TBC, pero no sea posible confirmar el diagnóstico mediante histopatología o exámenes de laboratorio, se puede realizar una prueba terapéutica administrando el tratamiento antifímico convencional, con el que se espera observar una respuesta favorable al mismo. ${ }^{8}$

Con respecto a las tuberculídes, es importante mencionar que por lo general es difícil detectar la presencia de M. tuberculosis con tinción de Ziehl-Neelsen o cultivo de Löwenstein-Jensen en el tejido afectado, pero sí puede observarse en sitios extracutáneos infectados por la micobacteria. Los pacientes desarrollan una reactividad fuerte a la tuberculina y son IGRA positivos. En la histopatología se observa inflamación granulomatosa. Las lesiones se resuelven con el tratamiento antifímico. ${ }^{8}$

Aunque el bacilo no se observa en el estudio histopatológico de las lesiones, sí se ha detectado ADN de $M$. tuberculosis en pacientes con tuberculídes papulonecróticas y con eritema indurado de Bazin, lo cual apoya el papel de la micobacteria en la patogénesis de las tuberculídes. En un estudio reciente que empleó PCR habitual, seguida de PCR anidada, logró aislarse ADN de M. tuberculosis en $80 \%$ de las muestras con diagnóstico de eritema indurado de Bazin analizadas, y en $80 \%$ de todas las muestras analizadas, $60 \%$ de ellas correspondían a tuberculídes. Los autores concluyen que esta técnica mejora la sensibilidad para detectar el ADN de la micobacteria, en particular, en el caso de las tuberculídes. ${ }^{9}$

El tratamiento de la TBC es igual al de la tuberculosis pulmonar, se sigue la estrategia TAES (tratamiento acortado estrictamente supervisado): fase intensiva (dos meses de tratamiento con rifampicina $600 \mathrm{mg} /$ día, isoniazida $300 \mathrm{mg} /$ día, pirazinamida $1,500 \mathrm{mg} / \mathrm{día}$ y etambutol $1,000 \mathrm{mg} / \mathrm{día}$ ), seguida de la fase de mante- 
nimiento durante cuatro meses con rifampicina $600 \mathrm{mg} /$ día e isoniazida $600 \mathrm{mg} / \mathrm{día}^{10}{ }^{10}$ Sin embargo, se sugiere que para el eritema indurado de Bazin se prolongue el tratamiento con isoniazida hasta por dos años. ${ }^{5,11}$

\section{CASO CLÍNICO}

Paciente del sexo femenino, de 45 años de edad, quien acude al Centro Dermatológico Pascua por presentar dermatosis diseminada a extremidades inferiores, en las que afectaba cara posterior de piernas en su tercio medio e inferior, bilateral con tendencia a la simetría; constituida por cuatro nódulos eritematosos, dolorosos a la palpación. De evolución crónica (Figura 1).

Al interrogatorio refería un mes de evolución con las lesiones. Además, la paciente informó que su madre fue diagnosticada con tuberculosis pulmonar hacía 12 años, y ella contaba con el antecedente de haber sido diagnosticada con tuberculosis cutánea variedad nodular profunda un año antes, fue tratada con la estrategia TAES con DOTBAL en su centro de salud durante seis meses. Cursó con buena evolución, con desaparición de dichas lesiones al mes de iniciar el tratamiento; sin embargo, reaparecieron dos semanas después de terminar dicho esquema.

Con la sospecha diagnóstica de recurrencia de TBC se inicia plan de estudio. Se solicitaron: PPD (derivado

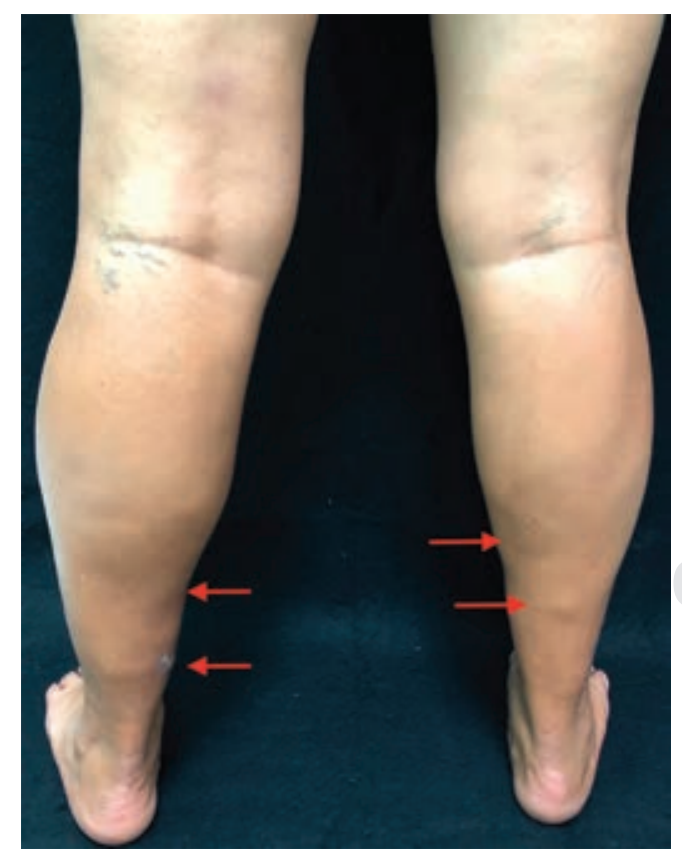

Figura 1: Nódulos diseminados.

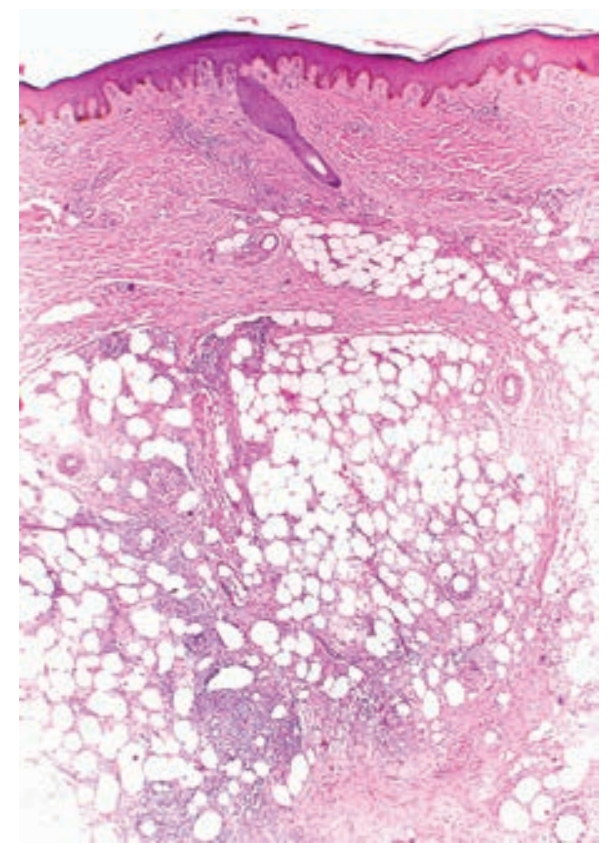

Figura 2: Paniculitis mixta granulomatosa con daño vascular (H\&E 40x).

proteico purificado), el cual no desarrolló induración; radiografía de tórax, la cual se encontraba sin alteraciones; bacilos acidorresistentes (BAAR) en orina tres muestras negativas, y exámenes de laboratorio que fueron reportados con las siguientes cifras: glucosa $128 \mathrm{mg} / \mathrm{dL}$, nitrógeno de urea en sangre $12.3 \mathrm{mg} / \mathrm{dL}$, creatinina 0.6 $\mathrm{mg} / \mathrm{dL}$, colesterol total $141 \mathrm{mg} / \mathrm{dL}$, triglicéridos $209 \mathrm{mg} / \mathrm{dL}$, hemoglobina $12.5 \mathrm{~g} / \mathrm{dL}$, plaquetas $250,000 / \mu \mathrm{L}$, leucocitos $5,700 / \mu \mathrm{L}$, neutrófilos $3,260 / \mu \mathrm{L}$, linfocitos $2059 / \mu \mathrm{L}$.

Se realizó nueva biopsia de uno de los nódulos. La imagen histopatológica mostró moderados infiltrados linfohistiocitarios perivasculares y perianexiales en dermis superficial y media. En dermis profunda y tejido celular subcutáneo, tanto en la porción septal como en la lobulillar, se apreciaban moderados infiltrados en focos constituidos por linfocitos, histiocitos, células epitelioides, esbozos de células gigantes multinucleadas, células gigantes tipo Langhans y de tipo cuerpo extraño. Dicho infiltrado invadía algunos vasos (Figura 2). Con lo anterior se realizó el diagnóstico histopatológico de paniculitis mixta granulomatosa con daño vascular.

Al tener en cuenta los antecedentes (combe positivo, biopsia previa con diagnóstico de paniculitis lobulillar granulomatosa que respondió adecuadamente a tratamiento antifímico), se consideró que la paciente cursaba con una recurrencia de tuberculosis cutánea variedad nodular profunda. Se le citó para continuar su 
evaluación e iniciar el tratamiento acorde al caso; sin embargo, hasta la fecha no ha acudido.

\section{DISCUSIÓN}

Por lo general, la TBC y las tuberculídes remiten con el tratamiento antifímico estándar recomendado por la OMS; sin embargo, el tratamiento del eritema indurado de Bazin puede requerir de más tiempo. Se ha propuesto la administración de isoniazida $400 \mathrm{mg} / \mathrm{día}$ como terapia de mantenimiento hasta por dos años, así como añadir piridoxina para prevenir la aparición de neuropatía periférica por su ingesta. El empleo de otros adyuvantes (dapsona, yoduro de potasio, doxiciclina y corticosteroides) ha demostrado resultados

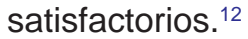

En las guías de Brasil 2011 para el tratamiento de la tuberculosis, los expertos recomiendan que para pacientes que necesiten retratamiento se debe iniciar nuevamente con el régimen estándar hasta que se cuente con los resultados de los cultivos y pruebas de sensibilidad con los fármacos administrados. Aquellos casos que no mejoren deben analizarse minuciosamente, verificando los medicamentos empleados, la adherencia y la evidencia de una posible resistencia a fármacos. ${ }^{13}$

Las guías del CDC (Center for Disease Control and Prevention) mencionan que en caso de sospechar falla al tratamiento (definida como la presencia de cultivos positivos después de cuatro meses de tratamiento antituberculoso) o recaída (definida como tuberculosis recurrente en cualquier momento después de haber completado tratamiento con curación), deberá aislarse M. tuberculosis para realizar pruebas de sensibilidad a fármacos de primera y segunda línea de tratamiento..$^{14,15}$

La recaída puede ocurrir como resultado de reinfección por la misma cepa (más frecuente en lugares con baja incidencia) o debido a reinfección exógena con una nueva cepa (más frecuente en lugares de alta incidencia). La mayoría de las recaídas se desarrollan dentro los primeros 6-12 meses después de haber completado el tratamiento, y en general son ocasionadas por micobacterias susceptibles. ${ }^{16}$

El tratamiento de tuberculosis cutánea farmacorresistente suele ser difícil y requiere el empleo de fármacos de segunda línea y/o resección quirúrgica. La farmacorresistencia se clasifica en: ${ }^{17}$

Monorresistencia: tuberculosis causada por cepas de $M$. tuberculosis resistentes in vitro a un medicamento de primera línea.
Polirresistencia: tuberculosis causada por cepas de $M$. tuberculosis resistentes in vitro a más de un medicamento de primera línea, menos a la isoniacida y rifampicina simultáneamente.

Multifarmacorresistencia (TB-MFR): tuberculosis causada por cepas de $M$. tuberculosis resistentes in vitro, como mínimo a la isoniacida y a la rifampicina, simultáneamente.

Resistencia extendida (TB-XFR): resistencia a isoniazida y rifampicina (MFR), más una quinolona, y uno o más de los tres inyectables de segunda línea (kanamicina, amikacina y capreomicina).

Los fármacos de segunda línea para tuberculosis farmacorresistente son: moxifloxacino $800 \mathrm{mg}$, clofazimina $100 \mathrm{mg}$, etambutol $1,200 \mathrm{mg}$, pirazinamida $2,000 \mathrm{mg}$, isoniazida $600 \mathrm{mg}$, protionamida $750 \mathrm{mg}$ y kanamicina máximo $1 \mathrm{~g} /$ día, inicialmente de cinco a siete días por semana (misma dosis que la amikacina). ${ }^{17}$

A partir del año 2016 la OMS recomienda que los esquemas con los fármacos arriba señalados tengan una duración de nueve a 12 meses.

La detección de la tuberculosis farmacorresistente requiere confirmación bacteriológica y pruebas de farmacorresistencia mediante pruebas moleculares específicas (que son muy rápidas), métodos de cultivo o tecnologías de secuenciación. ${ }^{17}$

La prueba Xpert MTB/RIF (Cepheid) es un estudio de PCR en tiempo real que puede detectar $M$. tuberculosis y resistencia a rifampicina en menos de dos horas, con una sensibilidad de $95 \%$ y una especificidad de $98 \% .^{18}$ Supone un avance importante en la detección precoz de este padecimiento. Se solicita en lugar de las pruebas convencionales (baciloscopía, cultivo y pruebas de farmacosensibilidad) como prueba diagnóstica inicial en adultos con tuberculosis asociada con $\mathrm{VIH}$, o por sospecha de cursar con tuberculosis multirresistente, y en los siguientes grupos de riesgo: fracasos a retratamiento primario y crónicos, contacto de un caso de tuberculosis multirresistente, fracaso a tratamiento primario, caso de tuberculosis con baciloscopía positiva al final del segundo o tercer mes de tratamiento primario o retratamiento, recaídas y reingresos por abandono, o en pacientes con tuberculosis y diabetes mellitus descompensada. Se realiza en muestras específicas (ganglio linfático y otros tejidos) procedentes de pacientes con sospecha de tener tuberculosis extrapulmonar.

Otra técnica molecular es el GenoType MDRplus (Hain), o ensayo de prueba en línea (Line Probe Assay), el cual puede detectar simultáneamente mutaciones en los genes que codifican resistencia a isoniacida y 
rifampicina en un plazo de 6-24 horas. Ambas pruebas pueden realizarse en forma directa sin que sea necesario esperar al crecimiento en un cultivo.

En el caso de la paciente, motivo de esta publicación, desconocemos si emplear isoniazida hasta por dos años, después de haber finalizado con el tratamiento estandarizado por la OMS, disminuiría su riesgo de una nueva recurrencia. En ella estaba indicado realizar pruebas de farmacosensibilidad (antes de reiniciar con la estrategia TAES), y en caso de no encontrar resistencia, iniciar retratamiento primario por ocho meses con isoniazida, rifampicina, pirazinamida, etambutol y estreptomicina por dos meses; seguido de isoniazida, rifampicina, pirazinamida y etambutol por un mes; y finalmente isoniazida, rifampicina y etambutol por cinco meses. Si hubiese desarrollado resistencia al menos a isoniazida y pirazinamida, lo indicado hubiese sido iniciar tratamiento estandarizado de segunda línea. ${ }^{17}$

Existen pocos datos en la literatura acerca de la recurrencia de la tuberculosis cutánea posterior a tratamiento, ya con curación clínica. En un estudio de seguimiento durante cinco años se encontró que $12.5 \%$ de los pacientes con diagnóstico de eritema indurado de Bazin desarrollaron recurrencia posterior a recibir el tratamiento antifímico estándar. ${ }^{12}$

\section{COMENTARIO}

Aunque la mayoría de los pacientes con tuberculosis cutánea responden adecuadamente al tratamiento estandarizado recomendado por la OMS, se desconoce hasta el momento el porcentaje de recurrencia de las diferentes variedades clínicas, por lo que se necesitan estudios de seguimiento a largo plazo que evalúen la recurrencia con el tratamiento convencional y la respuesta a los fármacos de segunda línea.

\section{REFERENCIAS}

1. van Zyl L, du Plessis J, Viljoen J. Cutaneous tuberculosis overview and current treatment regimens. Tuberculosis (Edinb). 2015; 95: 629-638.

2. Melsew YA, Doan TN, Gambhir M, Cheng AC, McBryde E, Trauer JM. Risk factors for infectiousness of patients with tuberculosis: a systematic review and meta-analysis. Epidemiol Infect. 2018; 146: 345-353.

3. Suárez Castañón C, Pérez Méndez C, Alvarez Cuesta CC, Corrales Canel B, Blanco Costa Ml. Erythema induratum of Bazin associated to Mycobacterium tuberculosis infection. Arch Argent Pediatr. 2012; 110: e43-e46.

4. Rodríguez O. Tuberculosis cutánea. Rev Fac Med UNAM. 2003; 46: 157-161.
5. Bravo F, Gotuzzo E. Cutaneous tuberculosis. Clin Dermatol. 2007; 25: $173-180$.

6. Calonje JE, Brenn T, Lazar A, Billings S. McKee's pathology of the skin. 4th edition. New York: Elsevier; 2012. pp. 346-349.

7. Chakrabortty R, Rahman MA, Ferdousi KR, Paul BK. Cutaneous tuberculosis: an update. Mymensingh Med J. 2016; 25: 385-391.

8. Tirado-Sánchez A, Bonifaz A. Cutaneous tuberculosis: a review of the current literature. Curr Trop Med Rep. 2018; 5: 67-76.

9. Maldonado-Bernal C, Ramos-Garibay A, Rios-Sarabia N, Serrano H, Carrera M, Navarrete-Franco G et al. Nested polymerase chain reaction and cutaneous tuberculosis. Am J Dermatopathol. 2019; 41: 428-435.

10. Secretaría de Salud. Programa de acción específico. Tuberculosis. 2013-2018. México: 2018. pp. 11-42.

11. Nirmala C, Nagarajappa N. Erythema induratum. A type of cutaneous tuberculosis. Indian J Tuberc. 2010; 57: 160-164.

12. Chen $Q$, Chen $W$, Hao F. Cutaneous tuberculosis: a great imitator. Clin Dermatol. 2019; 37: 192-199.

13. Brasil. Ministério da Saúde. Secretaria de Vigilância em Saúde. Departamento de Vigilância Epidemiológica. Manual de recomendações para o controle da tuberculose no Brasil. [Ministry of Health. Secretary of Health Surveillance. Department of Epidemiological Surveillance. Guide: recommendations for tuberculosis control in Brazil] Brasília: Ministério da Saúde; 2011. p. 284.

14. Centers for Disease Control and Prevention. Tuberculosis: laboratory information. [Accessed on June 16, 2016] Available in: http://www. cdc.gov/tb/topic/laboratory/

15. Centers for Disease Control and Prevention. Reference Laboratory Division of TB Elimination Laboratory User Guide for U.S. Public Health Laboratories: molecular detection of drug resistance (MDDR) in Mycobacterium tuberculosis complex by DNA sequencing (Version 2.0), June 2012. CDC, Atlanta, GA 2012. [Accessed on June 16, 2016] Available in: http://www.cdc.gov/tb/topic/laboratory/ mddrusersguide.pdf

16. Marx FM, Dunbar R, Enarson DA, Williams BG, Warren RM, van der Spuy GD et al. The temporal dynamics of relapse and reinfection tuberculosis after successful treatment: a retrospective cohort study. Clin Infect Dis. 2014; 58: 1676-1683.

17. Secretaría de Salud. Guía para la atención de personas con tuberculosis resistente a fármacos. Disponible en: http://www. cenaprece.salud.gob.mx/programas/interior/micobacteriosis/ descargas/pdf/guia_tb_mfr_ok.pdf

18. World Health Organization. Using the Xpert MTB/RIF assay to detect pulmonary and extrapulmonary tuberculosis and rifampicin resistance in adults and children: expert group meeting report: 2013. Geneva: World Health Organization.

Correspondencia:

Dr. Miguel Ángel Cardona-Hernández

Dr. Vértiz Núm. 464, esq. Eje 3 Sur,

Col. Buenos Aires, 06780,

Alcaldía Cuauhtémoc, CDMX.

Tel: 55 5519-6351

E-mail: drmiguelcardona08@gmail.com 